\title{
Fluorocarbon vapors slow down Coalescence in Foams
}

Katja Steck ${ }^{1, *}$, Martin Hamann ${ }^{1}$, Sébastien Andrieux ${ }^{1}$, Pierre Muller ${ }^{1}$, Patrick Kékicheff $^{1}$, Cosima Stubenrauch ${ }^{2,3}$, Wiebke Drenckhan ${ }^{1}$

${ }^{1}$ Institut Charles Sadron, University of Strasbourg, CNRS UPR22, 23 Rue du Loess, 67037 Strasbourg, France

${ }^{2}$ Institute of Physical Chemistry, University of Stuttgart, Pfaffenwaldring 55, 70569 Stuttgart, Germany

${ }^{3}$ Institute of Advanced Studies (USIAS), University of Strasbourg, F-67000 Strasbourg, France

\begin{abstract}
Controlling the stability of liquid foams is of the utmost importance for a wide range of applications. For decades, fluorocarbon vapors have been added to the gas phase of foams to ensure long-term stability against bubble coarsening. However, we show here for the first time that they also have an unexpected and pronounced effect on bubble coalescence. We quantify this effect in detail for foams stabilized by the nonionic surfactant hexaethylene glycol monododecyl ether $\left(\mathrm{C}_{12} \mathrm{E}_{6}\right)$ at controlled fluorocarbon (here: perfluorohexane $\left.\left(\mathrm{C}_{6} \mathrm{~F}_{14}\right)\right)$ concentrations, but we observe it for all investigated surfactants. Measuring surface tensions of the foaming solution with increasing fluorocarbon concentration, we found a synergy between the fluorocarbon and the surfactant which can be explained by (a) the formation of mixed layers of both species at the gas/water interface at low fluorocarbon concentrations and (b) the formation of a macroscopic fluorocarbon film if the gas phase is saturated with fluorocarbon vapor. The precise mechanism responsible for reducing coalescence, i.e. film rupture, remains to be elucidated.
\end{abstract}

\section{Article}

\section{Introduction}

Liquid foams are omnipresent in a vast range of applications ${ }^{[1-4]}$ : food and beverages, cosmetics, medicine, cleaning, firefighting, or as templates for solid foams. One major challenge in optimizing liquid foams for applications is to tune their stability, which is controlled by three main mechanisms: gravity-driven "drainage" of liquid, "coalescence" (rupture of films) and "coarsening" (gas transport between bubbles of different Laplace pressures). By carefully combining appropriate foaming methods and formulations (stabilizing agents, viscosity of the solution, or the gas composition), these destabilization mechanisms can be reduced or even fully stopped $^{[1-4]}$. Coarsening, for instance, is commonly prevented by adding fluorocarbon vapors to the foaming gas ${ }^{[1,4]}$. Since fluorocarbons are quasi insoluble in water ${ }^{[5]}$, their transport through the aqueous films separating neighboring bubbles is hampered. As a result, an osmotic pressure difference between neighboring bubbles is created, which counteracts the destabilizing Laplace 
pressure difference. Fluorocarbon vapors are thus widely used in foam science and biomedical applications, e.g., to stabilize microbubbles ${ }^{[6-9]}$.

However, until now, fundamental and applied research completely neglected the possible influence of fluorocarbon vapors on other foam properties in general, and on foam coalescence in particular. We show here that fluorocarbon vapors can very effectively hinder coalescence even at very low concentrations. We hypothesize that this is due to the formation of a mixed fluorocarbon/surfactant layer at the gas/water interface. The complex properties of surfactant monolayers in the presence of fluorocarbons have been investigated in depth in recent years ${ }^{[10-15]}$, yet without establishing a link to foam properties. Our findings open new possibilities of controlling foam stability by introducing a "co-adsorbate" from the gas phase.

\section{Materials and methods}

To quantify the effect of fluorocarbon vapors on foam coalescence we investigated foams stabilized by aqueous solutions of the nonionic surfactant hexaethylene glycol monododecylether $\left(\mathrm{C}_{12} \mathrm{E}_{6}\right)$ at different concentrations of perfluorohexane in the gas $\left(\mathrm{N}_{2}\right)$. We chose $\mathrm{C}_{12} \mathrm{E}_{6}$ as its foams are highly unstable, with coalescence being the determining destabilization mechanism ${ }^{[16-18]}$. The solutions were prepared at a $\mathrm{C}_{12} \mathrm{E}_{6}$ concentration of $c=10 \mathrm{cmc}\left(\mathrm{cmc}=0.8 \cdot 10^{-4} \mathrm{M}\right)$ using Milli-Q water and sodium chloride $(\mathrm{NaCl})$ at $10^{-2} \mathrm{M}$. NaCl is required to ensure the conductivity of the solution to measure the foam's liquid fraction (liquid volume divided by foam volume), but it does not affect foam stability (Figure $\mathrm{S} 1$ in Supplementary Materials). $\mathrm{C}_{12} \mathrm{E}_{6}$ and perfluorohexane were purchased from Sigma Aldrich and used without further purification. $\mathrm{NaCl}$ was cured overnight $\left(T=500{ }^{\circ} \mathrm{C}\right)$ to remove organic contaminants.

The foams were characterized by image analysis and conductivity measurements; (Section S2 in Supplementary Materials) with the commercially available FoamScan (http://teclis.eu). The fluorocarbon concentration in the gas was set by controlling the partial pressure $p$ of perfluorohexane in $\mathrm{N}_{2}$ with respect to its vapor pressure $p_{0}$ (taken to be $24 \mathrm{kPa}$ at $20{ }^{\circ} \mathrm{C}^{[19]}$ ). The ratio $p / p_{0}$ was adjusted by adding different volumes of liquid perfluorohexane $(V=2 ; 7.5 ; 15 ; 30$ $\mu \mathrm{L}$ ) to $60 \mathrm{~mL}$ syringes already filled with $50 \mathrm{~mL}$ of $\mathrm{N}_{2}$ at atmospheric pressure (taken to be $10^{5}$ $\mathrm{Pa}$ ). With an immediate evaporation in the syringe, the final values correspond to $p / p_{0}=0.02,0.08$, 0.15 and 0.30 , respectively (calculated according to ${ }^{[20]}$ ) with an estimated error of $5 \%$. Using a syringe pump from Harvard Apparatus, the gas mixture was injected into the foaming solution through a porous glass frit, creating fine bubbles whose sizes depend on the pore sizes of the frit. Glass frits (Teclis) with two different average pore sizes $R_{\mathrm{f}}=5-8 \mu \mathrm{m}(\mathrm{P} 4)$ and 20-50 $\mu \mathrm{m}(\mathrm{P} 2)$ were used to obtain two different bubble sizes. Since the results are similar for both glass frits, we only discuss those obtained with the P4 glass frit. The results obtained with the P2 glass frit (larger bubbles) are shown in the Supporting Information (Figures S2 and S3 in Supplementary Materials). All foams were generated at room temperature $\left(T=20 \pm 1{ }^{\circ} \mathrm{C}\right)$ with a total gas volume of $100 \mathrm{~mL}$ and a flow rate of $80 \mathrm{~mL} \mathrm{~min}^{-1}$. Note that the foam column was closed with Parafilm to ensure that the foam is in contact with a gas mixture at the same $p / p_{0}$. The goal was to minimize the osmotic pressure difference with the exterior, which would lead to a net gas transport into the foam and hence its growth with time ${ }^{[21]}$. Only a small hole was left in the cover to allow for pressure equilibration. The first measurement was performed to ensure equilibrium conditions while the results of the second to fourth measurements were averaged for foam height and bubble 
size distributions. The measurements were highly reproducible as shown in Figure S4 of the Supplementary Materials.

\section{Results and discussion}

A selection of photographs of foams generated with the $\mathrm{P} 4$ glass frit (smaller bubbles) is shown in Figure 1a for three different values of $p / p_{0}$ at 60, 300 and $1000 \mathrm{~s}$ after foam generation. In pure $\mathrm{N}_{2}$ $\left(p / p_{0}=0\right)$ the foams rapidly collapse: their initial height is halved within the first $300 \mathrm{~s}$ after gas injection, and only a small portion of foam remains at $1000 \mathrm{~s}$. In contrast, the foams are much more stable when perfluorohexane is present in the gas phase $\left(p / p_{0}>0\right)$. At ratios as low as $p / p_{0}=0.02$, the foam height is constant over the first $300 \mathrm{~s}$ after gas injection and starts to decrease only at long time scales (here $\sim 1000 \mathrm{~s}$ ). However, higher $p / p_{0}$ allow the foam height $h$ to be even more stable as illustrated by plotting $h$ as a function of time for five different values of $p / p_{0}$ (Figure $2 \mathrm{a}$ ).

Since we are only interested in coalescence, we must rule out that coarsening happens simultaneously. The characteristic time scale of coarsening depends on the bubble size distribution, the liquid content of the foam, the stabilizing agent and the diffusing gas ${ }^{[1]}$. For the foams shown in Figure 1 we can estimate characteristic coarsening times of 100-1000s (Section S3 in Supplementary Materials), which is why in the following we only focus on the first $300 \mathrm{~s}$. Figure $1 \mathrm{~b}$ shows close-up photographs of the foam bubbles $60 \mathrm{~s}$ and $240 \mathrm{~s}$ after foam generation. The white zones are the parts of the bubbles which are pressed against the glass wall, while the black zones correspond to the liquid content of the foam which decreases with time due to gravitydriven drainage ${ }^{[22]}$. Its time-evolution (Figure S5 in Supplementary Materials) is directly related to the bubble size evolution in a well-known manner - larger bubbles lead to stronger drainage ${ }^{[1]}$ and will not be further discussed here. Using image treatment with ImageJ to relate the white zones to the bubble radii (protocol described in ${ }^{[18]}$ ), we can plot the bubble size distributions for different foam ages in Figure 1c. At $p / p_{0}=0$, one sees that the bubble size rapidly increases within the first $300 \mathrm{~s}$, as shown by a fast broadening of the corresponding bubble size distribution. Visual inspection of the foam confirms that this evolution is due to bubble coalescence. In the case of $p / p_{0}$ $>0$, the bubble size increases much more slowly $\left(p / p_{0}=0.02\right)$ or not at all $\left(p / p_{0}=0.30\right)$ within the time of the experiment. Moreover, the bubble size distributions remain narrow. 
(a) $p / p_{0}$

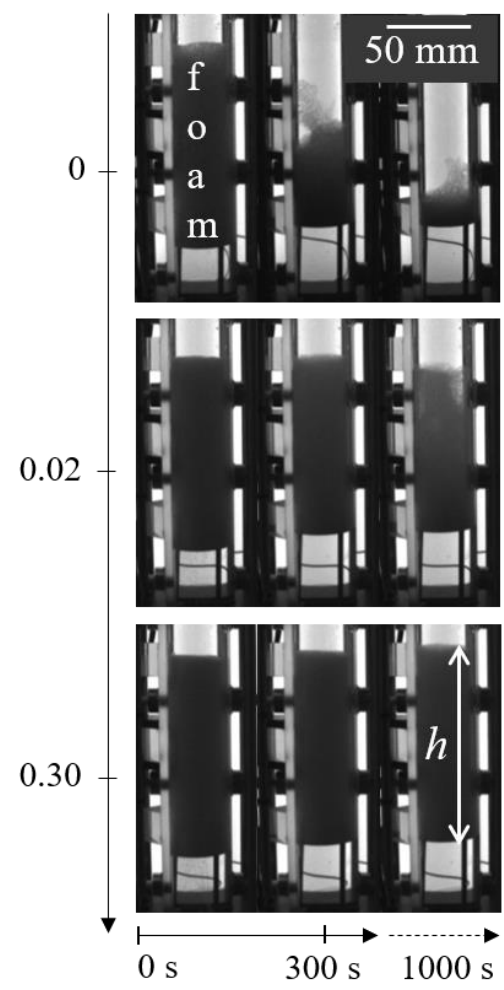

(b)

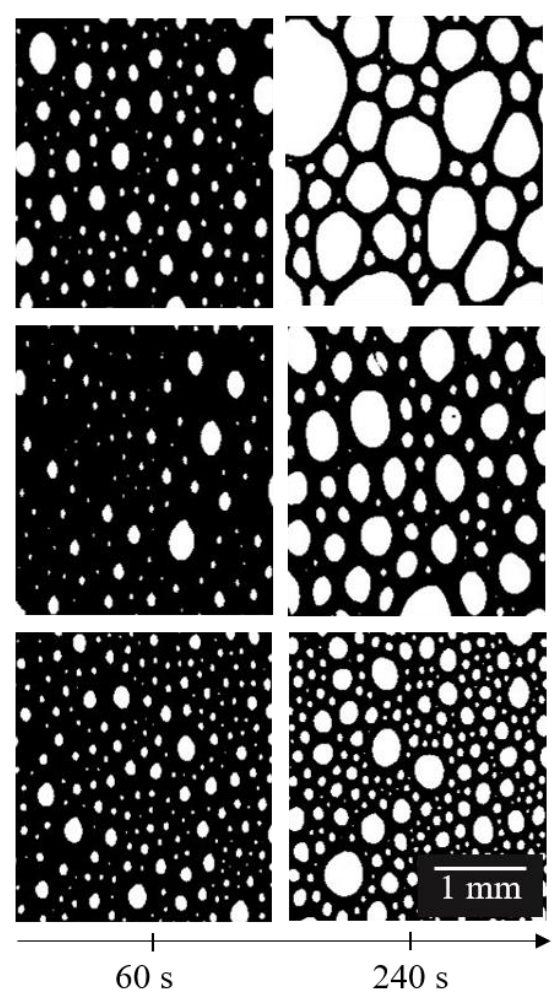

(c)

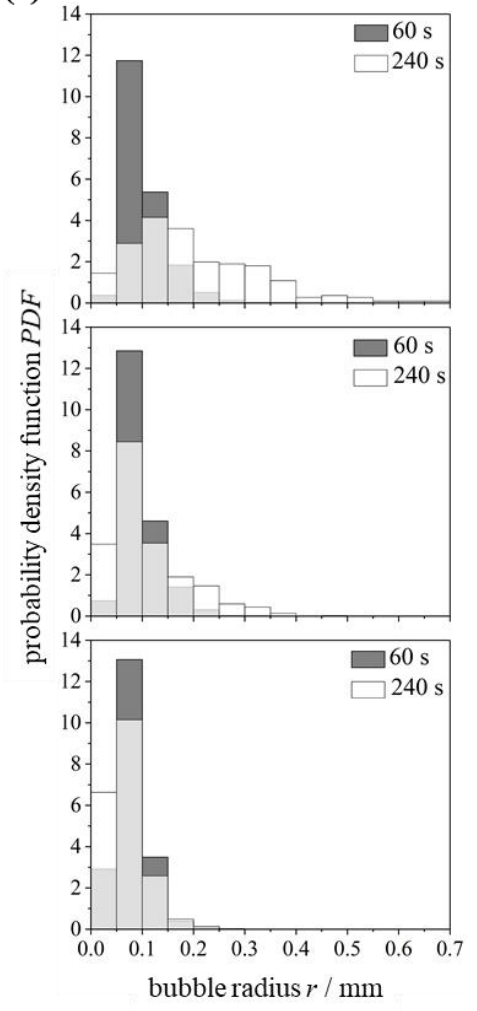

Figure 1: Evolution of foams of aqueous solutions of $\mathrm{C}_{12} \mathrm{E}_{6}$ at different ratios of partial pressure $p$ to vapor pressure $p_{0}$ of perfluorohexane in $\mathrm{N}_{2}$ : (a) Photographs of the foams and (b) close-up photographs of the bubbles in the foam generated in the FoamScan with a P4 frit. The white zones correspond to the part of the bubbles squeezed against the glass wall which can be related to the bubble size by image treatment. (c) Corresponding bubble size distributions (probability density functions $-P D F)$.

Figure $2 \mathrm{~b}$ shows the time-evolution of the mean bubble radius $\langle r\rangle$ for the same $p / p_{0}$-ratios as in Figure 2a, confirming the trends indicated in Figures $1 \mathrm{~b}$ and 1c: a rapid increase of the average bubble radius for $p / p_{0}=0$, a slow increase for $p / p_{0}=0.02$ and a constant bubble radius for $p / p_{0}>$ 0.02 .

In line with literature data ${ }^{[18]}$, the degradation characteristics of the foams at $p / p_{0}=0$ (rapid collapse of the foam; fast increase of the mean bubble radius; fast broadening of the bubble size distribution) indicate that coalescence is the main destabilization mechanism in the first $300 \mathrm{~s}$. However, it is very effectively slowed down in the presence of perfluorohexane vapor even at very low concentrations. While the foams generated at $p / p_{0}=0.02$ show a somewhat intermediate behavior, foams generated at $p / p_{0}>0.02$ are very stable, i.e. the fluorocarbon fully suppresses coalescence in the first $300 \mathrm{~s}$. 


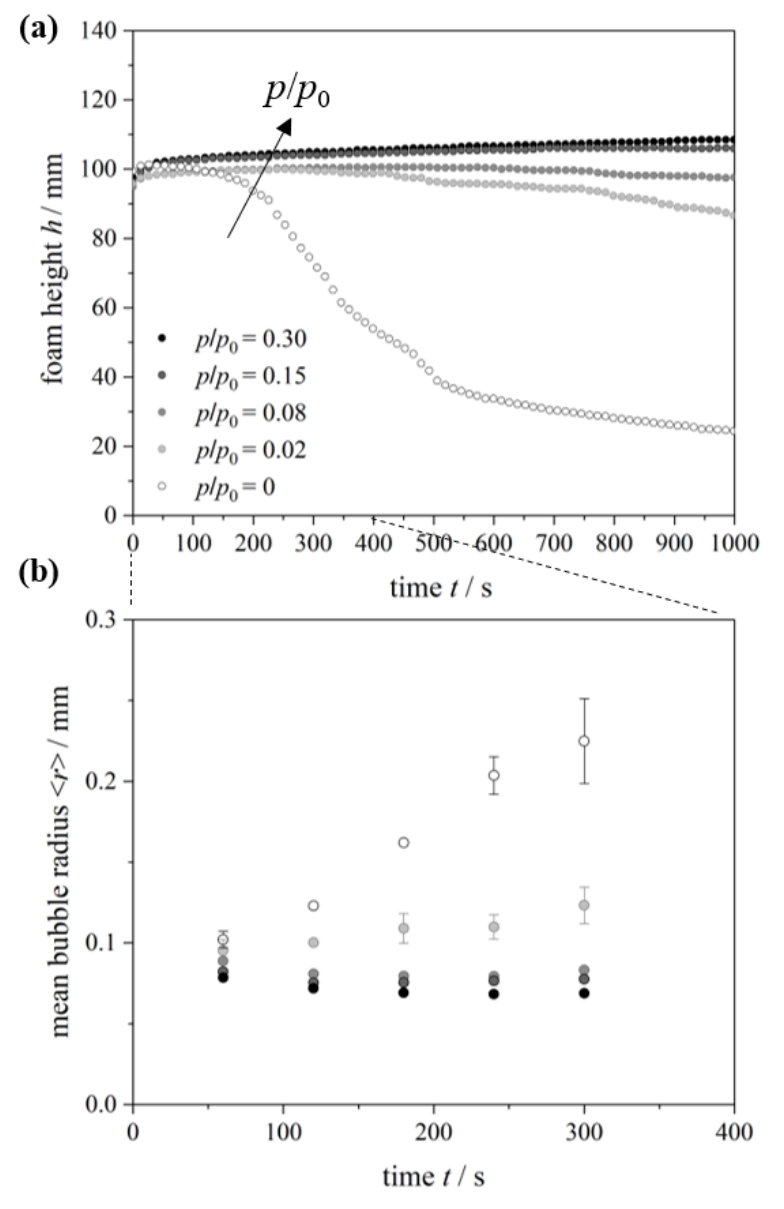

Figure 2: Evolution of the (a) foam height $h$ and (b) mean bubble radius $\langle r\rangle$ of aqueous solutions of $\mathrm{C}_{12} \mathrm{E}_{6}$ at different $p / p_{0}$-ratios using the same protocol as in Figure 1.

While the influence of fluorocarbon vapors on coarsening is well understood ${ }^{[1-4,23,24]}$, its pronounced influence on coalescence has not been studied yet. To obtain more insight, we measured the surface tension of the foaming solutions as a function of $p / p_{0}$ using the Tracker from Teclis (http://teclis.eu) (4 $\mu \mathrm{L}$ rising bubble in $25 \mathrm{~mL}$ surfactant solution). The gas mixtures were prepared as described for foaming. For each $p / p_{0}$, two series of measurements were recorded using 4 bubbles each time: the first bubble was used for system equilibration, whereas the results of the final three were averaged. In each measurement, the surface tension drops rapidly to its equilibrium value (a few seconds) in a highly reproducible manner (Figure S7 in Supplementary Materials). The spread of the data gives an average deviation of $\pm 0.2 \mathrm{mN} / \mathrm{m}$ for the surface tension.

Figure 3a shows, independently on whether air or pure $\mathrm{N}_{2}$ was used, that the equilibrium surface tension $\gamma$ of the $\mathrm{C}_{12} \mathrm{E}_{6}$ solution decreases measurably in the presence of perfluorohexane. Similar observations have already been reported for water ${ }^{[25,26]}$ and aqueous solutions of various surfactants such as cationic ones ${ }^{[11]}$, phospholipids ${ }^{[10,12,14]}$, and polyethylene glycols $\left(\mathrm{C}_{\mathrm{i}} \mathrm{E}_{\mathrm{j}}\right)^{[11]}$. They are attributed to the co-adsorption of perfluorohexane and the surfactant at the gas/water interface $^{[10-13]}$, forming a mixed layer at the interface of surfactants incorporating fluorocarbon molecules, as sketched in Figure 3b. Interestingly, in our system, the surface tension decreases 
linearly with increasing $p / p_{0}$. Although this observation awaits to be rationalized, we hypothesize that the formation of an initially mixed monolayer of perfluorohexane and surfactant at the gas/water interface at low ratios of $p / p_{0}$ is followed by the condensation of liquid perfluorohexane with increasing $p / p_{0}$ until a macroscopic film of pure liquid perfluorohexane is formed at $p / p_{0}=1$ (Figure 3b). This is supported by our finding that the surface tension at $p / p_{0}=1(\gamma=29.4 \mathrm{mN} / \mathrm{m})$ is very close to the sum of the surface tensions of the liquid perfluorohexane interface with the gas $(\gamma=13.0 \mathrm{mN} / \mathrm{m})^{[27]}$ and with the surfactant solution $(\gamma=16.6 \mathrm{mN} / \mathrm{m})$. This implies that the condensed film is thick enough so that its two bounding interfaces do not interact. Layers of liquid fluorocarbons have already been reported at solid and liquid surfaces ${ }^{[28,29]}$, e.g., smectic fluorocarbon layers can form at the water surface ${ }^{[29]}$ and are frequently observed in cloaked droplets on lubricant-infused surfaces ${ }^{[30,31]}$. In addition, similar observations have been described for the water surface and surfactant layers in contact with saturated alkane vapors ${ }^{[32-34]}$.

Future work needs to characterize how the film thickness depends on the FC $p / p_{0}$ using X-ray or neutron reflectivity, ellipsometry and/or fluorescence microscopy. However, since our work shows that the formation of a macroscopic film of pure perfluorohexane is not necessary for the suppression of coalescence, we concentrate on the lower range of $p / p_{0}\left(0.02 \leq p / p_{0}<0.3\right)$ which is sufficient to stabilize foams against coalescence.

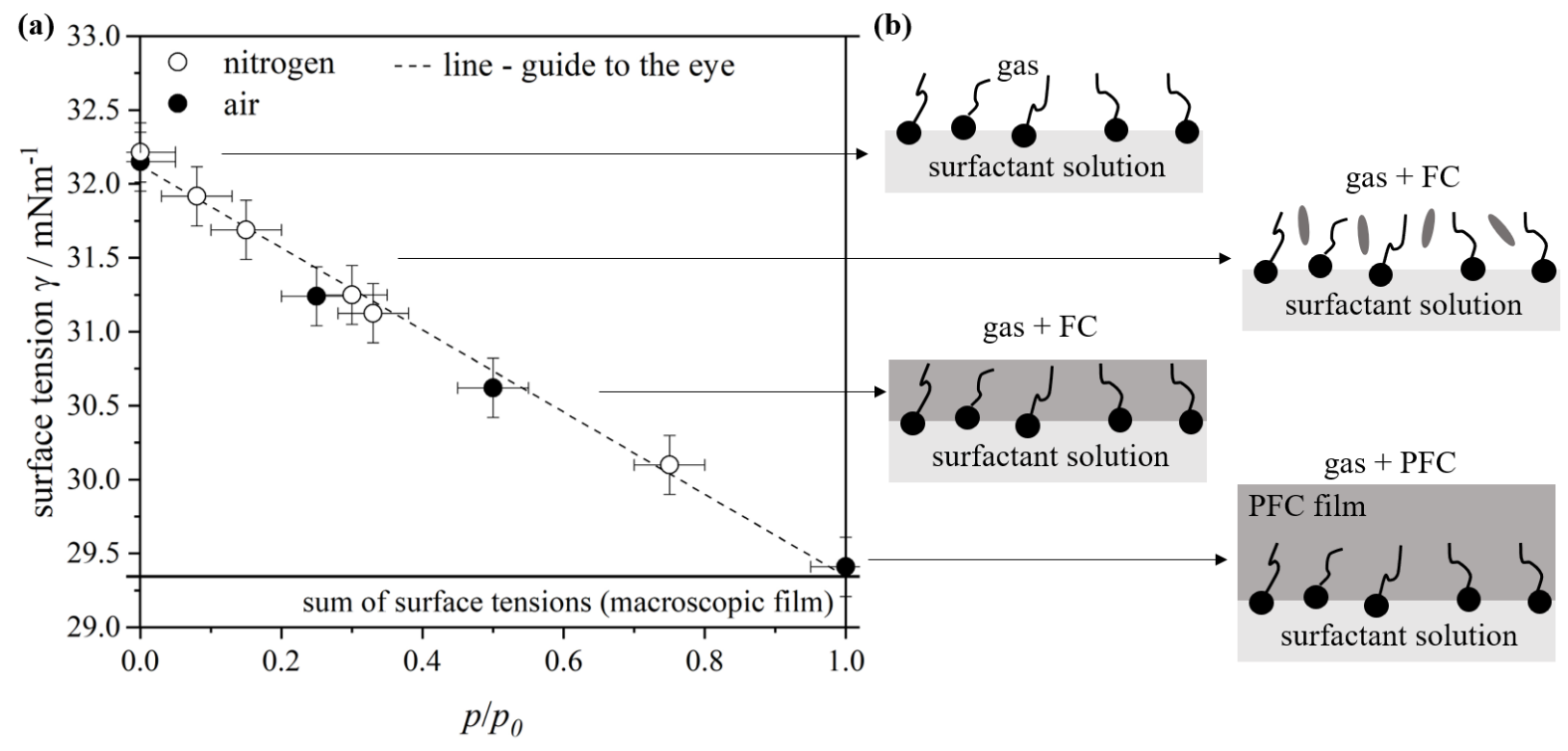

Figure 3: (a) Surface tension $\gamma$ of aqueous solutions of $\mathrm{C}_{12} \mathrm{E}_{6}$ at different ratios of partial pressure $p$ to vapor pressure $p_{0}$ of perfluorohexane in $\mathrm{N}_{2}$ (white circles) or air (black circles) at room temperature. (b) Hypothetic sketches of mixed fluorocarbon (FC)/surfactant layers of growing thickness with increasing $p / p_{0}$.

While a clear synergy is indicated by the decrease of surface tension with increasing fluorocarbon vapor concentration, its influence on foam coalescence still has to be elucidated in future studies. Reliable rationalization of foam coalescence - even for simple surfactant solutions - remains an 
open field ${ }^{[2,3,35-37]}$. It is known, however, that the surface tension alone is not sufficient to explain the ability of a surfactant to stabilize a foam. The formation of a mixed surfactant/fluorocarbon layer may influence film rupture in many different ways. For example, it may change the balance between the surface forces (for instance DLVO-like interactions) in the film if the Hamaker constant or/and the surface charge of the gas/water interface are affected ${ }^{[32,33]}$. It is important to note that in our case only the magnitude of the Hamaker constant can be affected and not its sign, since dispersion forces in symmetric films are always attractive ${ }^{[38]}$. Nevertheless, despite the fact that the effective profile and magnitude of the Hamaker constant are not only a function of the thicknesses of all involved media, but also of the screening by electrolytes, the possible changes in magnitude are rarely larger than a factor 2, as shown by the accurate numerical calculations carried out within the most advanced theoretical Lifshitz framework ${ }^{[38,39]}$. On the other hand, the damping of thermal fluctuations of the film thickness increases the effective Hamaker constant, but, at the same time, the entropy-driven repulsion (Helfrich repulsion-like type ${ }^{[40]}$ ) is increased, leading to a potentially more stable film. However, if heterogeneities occur at the interfaces, the picture may become very different ${ }^{[41,42]}$ : they will disrupt the symmetry between the two opposite surface films, at least at the scale of the lateral correlation length along these films. Such heterogeneities can arise from different effects ${ }^{[41-43]}$, including fluctuations in the surface charge distribution, polarization or dielectric properties, or curvature fluctuations of the monolayers. In either case it has been shown that these fluctuations ${ }^{[41-43]}$ can affect the long-range interactions which may contribute to enhanced (or conversely reduced) film stability.

Another important stabilizing factor may arise from the visco-elastic behavior of the mixed layer, which has been shown to be affected by the presence of fluorocarbons ${ }^{[10,14]}$. Interfacial viscoelasticity has an important influence on different dynamic phenomena associated with film rupture $^{[35]}$ : (1) It fixes the boundary conditions of the film drainage, and hence the characteristic drainage times. (2) It provides a self-healing elasticity which counteracts hole formation in the film. And (3) it has the capacity to dampen thermal fluctuations of the film thickness. In the past, it has been shown that fluorocarbons have the tendency to fluidify initially strongly visco-elastic monolayers made of lipids, polymers or proteins ${ }^{[10,11,14,44]}$. This could be very different for our surfactant monolayers whose dilational or shear visco-elastic moduli at such high concentrations are too small to be reliably characterized with classic techniques. In this case, the fluorocarbon could amplify the visco-elastic response and therefore slow down film drainage, increase selfhealing and dampen thermal fluctuations. Such a rigidification of the interface may arise from a "smectic-like" ordering of the perfluorohexane at the water surface, which was found by Kashimoto et al. ${ }^{[29]}$ using X-ray reflectivity measurements, and which could be even stronger in the presence of surfactants. This smectic-like ordering could create a "stabilizing shell" around the bubbles slowing down coalescence. We recall that a similar stabilizing mechanism is known for oil-based foams stabilized by lamellar layers (the two-component analogue to one-component smectic layers) formed around the bubbles ${ }^{[45-47]}$.

Another important interface-driven mechanism could be related to the energy required for hole formation in the film. An increasing number of investigations ${ }^{[48-50]}$ seem to confirm the statistical nature of the rupture of foam (and emulsion) films, with the probability of rupture being tightly linked to the energy required to create a hole in the film. Depending on the type of interface, this energy is dominated by surface tension, surface dilational and/or surface bending elasticity. In our 
case, the fluorocarbon may lead to a significant increase in this energy accompanied by a reduction in rupture probability.

Last but not least, the presence of a fluorocarbon film may also decrease water evaporation. However, reliable quantification of all these different effects is a major challenge and planned for future work.

\section{Conclusion and outlook}

In summary, we have shown - against common believes - that the presence of very small quantities of a fluorocarbon vapor (here: perfluorohexane) in the gas phase of liquid foams does not only slow down coarsening, but also coalescence. Hence, the long-term stability of foams with fluorocarbon vapors is not only due to the suppression of coarsening, but a combined effect including a reduction of coalescence. This is in contrast to similar investigations with alkane vapors, which decrease foam stability ${ }^{[51]}$ - a difference which needs to be dealt with in future studies. Surface tension measurements show that the influence of the fluorocarbon must arise from its co-adsorption with the surfactant at the gas/water interface, which was also observed for microbubbles $^{[44,52]}$, drops, or plain interfaces ${ }^{[26]}$. While the mechanisms that slow down coalescence await to be elucidated, our results clearly show that the fluorocarbon is a very efficient "coadsorbate" from the gas phase. Its adsorption leads to the formation of a mixed surfactantfluorocarbon layer which slows down coalescence.

For simplicity, we presented here detailed investigations of one surfactant only. Work on other surfactants is in progress and we can already state that our observations are valid for all the surfactants that we have tested so far. Figure 4 shows three representative examples, namely foams stabilized with the nonionic surfactant $\mathrm{C}_{12} \mathrm{DMPO}$ (ABCR Chemicals, $\mathrm{cmc}=3.1 \cdot 10^{-4} \mathrm{M}$ ), the cationic surfactant $\mathrm{C}_{14} \mathrm{TAB}$ (Sigma Aldrich, $\mathrm{cmc}=4.0 \cdot 10^{-3} \mathrm{M}$ ) both at $c \approx 10 \mathrm{cmc}$, and the amphiphilic siloxane-based polymer DBP-732 (Fluorochem) at a mass concentration beta $\approx 7 \mathrm{wt} \%$. Intensive work is in progress to quantify not only the influence of the type of surfactant, but also of the fluorocarbon on the stability of foams and isolated films. In the longer run it will be desirable to transfer the obtained understanding to hydrophobic vapors which are less harmful to the environment. However, the fact that alkane vapors have a similar influence on surface tension but an opposite influence on foam stability ${ }^{[51]}$, indicates that some intricate synergies await to be unraveled in this exciting field! 
(a) $\mathrm{C}_{12} \mathrm{DMPO}$

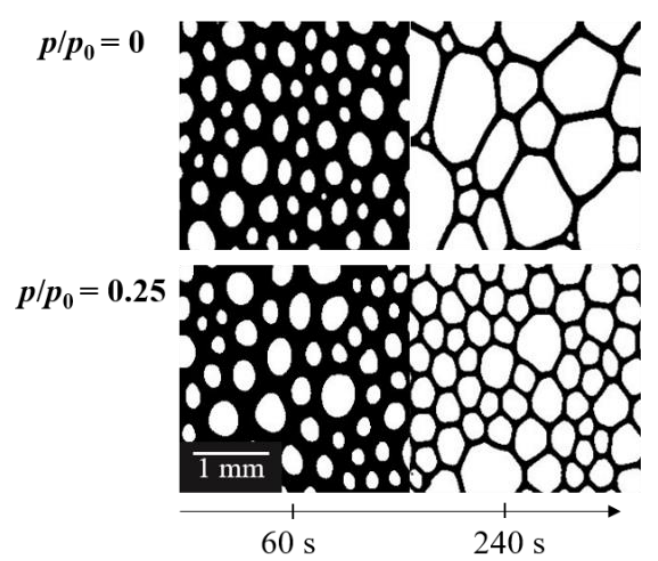

(b) $\mathrm{C}_{14} \mathrm{TAB}$
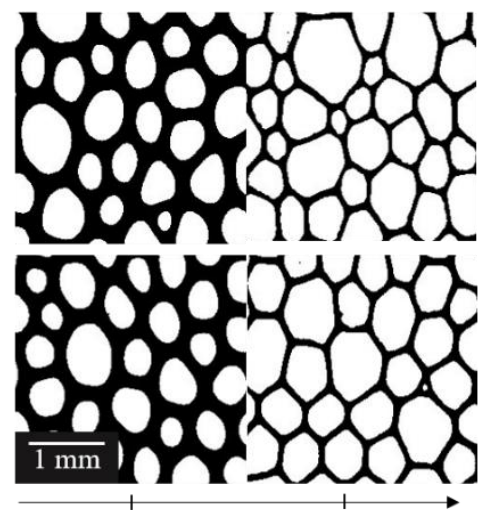

$60 \mathrm{~s}$

$240 \mathrm{~s}$
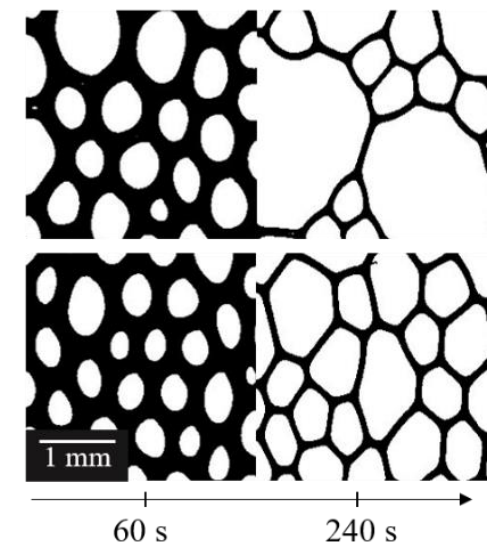

Figure 4: Evolution of foams of aqueous solutions of (a) nonionic dodecyldimethyl phosphineoxide $\left(\mathrm{C}_{12} \mathrm{DMPO}\right)$, (b) cationic tetradecyltrimethyl ammonium bromide $\left(\mathrm{C}_{14} \mathrm{TAB}\right)$ and (c) dimethylsiloxane block copolymer (DBP732) at different ratios of partial pressure $p$ to vapor pressure $p_{0}$ of perfluorohexane in air.

\section{Acknowledgement}

We would like to thank Tamara Schad, Dominique Langevin, Marie-Pierre Krafft, Elise Lorenceau and Céline Hadji for numerous stimulating discussions. We thank the internship students Hugo Barbier and Guillaume Cotte-Carluer for some preliminary investigations. Leandro Jacomine's and Natalie Preisig's help for many technical and organizational aspects is greatly acknowledged. Cosima Stubenrauch acknowledges a fellowship by the Institute of Advanced Studies at the University of Strasbourg (USIAS) and Wiebke Drenckhan acknowledges financial support by an ERC consolidator grant (agreement 819511 - METAFOAM).

\section{Conflict of Interest}

The authors declare no conflict of interest

Keywords foam stability, coalescence, fluorocarbon vapors, co-adsorption, foam film, film rupture 


\section{References}

[1] I. Cantat, S. Cohen-Addad, F. Elias, F. Graner, R. Höhler, O. Pitois, F. Rouyer, A. SaintJalmes, R. Flatman, Foams: Structure and Dynamics, Oxford University Press, 2013.

[2] D. Langevin, Emulsions, Microemulsions and Foams, Springer International Publishing, Cham, 2020.

[3] R. J. Pugh, Bubble and Foam Chemistry, Cambridge University Press, Cambridge, 2016.

[4] D. L. Weaire, S. Hutzler, The Physics of Foams, Clarendon Press, Oxford, 2001.

[5] J. G. Riess, Artif. Cells. Blood Substit. Immobil. Biotechnol. 2005, 33, 47-63.

[6] A. Kabalnov, J. A. Bradley, S. Flaim, D. Klein, T. Pelura, B. Peters, S. Otto, J. Reynolds, E. Schutt, J. Weers, Ultrasound Med. Biol. 1998, 24, 751-760.

[7] A. Kabalnov, D. Klein, T. Pelura, E. Schutt, J. Weers, Ultrasound Med. Biol. 1998, 24, 739-749.

[8] E. G. Schutt, D. H. Klein, R. M. Mattrey, J. G. Riess, Angew. Chemie Int. Ed. 2003, 42, 3218-3235.

[9] M. P. Krafft, J. Fluor. Chem. 2015, 177, 19-28.

[10] M. P. Krafft, V. B. Fainerman, R. Miller, Colloid Polym. Sci. 2015, 293, 3091-3097.

[11] V. B. Fainerman, E. V. Aksenenko, R. Miller, Adv. Colloid Interface Sci. 2017, 244, 100112.

[12] P. N. Nguyen, T. T. Trinh Dang, G. Waton, T. Vandamme, M. P. Krafft, ChemPhysChem 2011, 12, 2646-2652.

[13] X. Liu, C. Counil, D. Shi, E. E. Mendoza-Ortega, A. V. Vela-Gonzalez, A. Maestro, R. A. Campbell, M. P. Krafft, J. Colloid Interface Sci. 2021, 593, 1-10.

[14] F. Gerber, M. P. Krafft, T. F. Vandamme, M. Goldmann, P. Fontaine, Biophys. J. 2006, 90, 3184-3192.

[15] C. Hadji, B. Dollet, H. Bodiguel, W. Drenckhan, B. Coasne, E. Lorenceau, Langmuir 2020, 36, 13236-13243.

[16] C. Stubenrauch, L. K. Shrestha, D. Varade, I. Johansson, G. Olanya, K. Aramaki, P. Claesson, Soft Matter 2009, 5, 3070.

[17] C. Stubenrauch, P. M. Claesson, M. Rutland, E. Manev, I. Johansson, J. S. Pedersen, D. Langevin, D. Blunk, C. D. Bain, Adv. Colloid Interface Sci. 2010, 155, 5-18.

[18] J. Boos, W. Drenckhan, C. Stubenrauch, J. Surfactants Deterg. 2013, 16, 1-12.

[19] A. M. A. Dias, C. M. B. Gonçalves, A. I. Caço, L. M. N. B. F. Santos, M. M. Piñeiro, L. F. Vega, J. A. P. Coutinho, I. M. Marrucho, J. Chem. Eng. Data 2005, 50, 1328-1333.

[20] H. Bey, F. Wintzenrieth, O. Ronsin, R. Höhler, S. Cohen-Addad, Soft Matter 2017, 13, 6816-6830. 
[21] S. Andrieux, Monodisperse Highly Ordered and Polydisperse Biobased Solid Foams, Springer International Publishing, Cham, 2019.

[22] E. Forel, E. Rio, M. Schneider, S. Beguin, D. Weaire, S. Hutzler, W. Drenckhan, Soft Matter 2016, 12, 8025-8029.

[23] D. Weaire, V. Pageron, Philos. Mag. Lett. 1990, 62, 417-421.

[24] F. G. Gandolfo, H. L. Rosano, J. Colloid Interface Sci. 1997, 194, 31-36.

[25] D. Baumer, G. H. Findenegg, J. Colloid Interface Sci. 1982, 85, 118-127.

[26] V. S. Chernyshev, M. Skliar, Soft Matter 2014, 10, 1937-1943.

[27] M. G. Freire, P. J. Carvalho, A. J. Queimada, I. M. Marrucho, J. A. P. Coutinho, J. Chem. Eng. Data 2006, 51, 1820-1824.

[28] M. J. O’Keefe, J. M. Rigsbee, MRS Proc. 1993, 304, 179.

[29] K. Kashimoto, J. Yoon, B. Hou, C. H. Chen, B. Lin, M. Aratono, T. Takiue, M. L. Schlossman, Phys. Rev. Lett. 2008, 101, DOI 10.1103/PhysRevLett.101.076102.

[30] F. Schellenberger, J. Xie, N. Encinas, A. Hardy, M. Klapper, P. Papadopoulos, H.-J. Butt, D. Vollmer, Soft Matter 2015, 11, 7617-7626.

[31] Q. Ge, A. Raza, H. Li, S. Sett, N. Miljkovic, T. Zhang, ACS Appl. Mater. Interfaces 2020, $12,22246-22255$.

[32] A. Javadi, N. Moradi, V. B. Fainerman, H. Möhwald, R. Miller, Colloids Surfaces A Physicochem. Eng. Asp. 2011, 391, 19-24.

[33] V. B. Fainerman, E. V. Aksenenko, V. I. Kovalchuk, R. Miller, Colloids Surfaces A Physicochem. Eng. Asp. 2016, 505, 118-123.

[34] V. B. Fainerman, E. V. Aksenenko, V. I. Kovalchuk, A. Javadi, R. Miller, Soft Matter 2011, 7, 7860-7865.

[35] E. Rio, A.-L. Biance, ChemPhysChem 2014, 15, 3692-3707.

[36] D. Langevin, Curr. Opin. Colloid Interface Sci. 2015, 20, 92-97.

[37] D. Langevin, Curr. Opin. Colloid Interface Sci. 2019, 44, 23-31.

[38] J. Mahanty, B. W. Ninham, Dispersion Forces, Academic Press, London, New York, San Francisco, 1976.

[39] V. E. Shubin, P. Kékicheff, J. Colloid Interface Sci. 1993, 155, 108-123.

[40] W. Helfrich, Zeitschrift für Naturforsch. A 1978, 33, 305-315.

[41] P. Kékicheff, J. Iss, P. Fontaine, A. Johner, Phys. Rev. Lett. 2018, 120, 118001.

[42] P. Kékicheff, Adv. Colloid Interface Sci. 2019, 270, 191-215.

[43] V. Meklesh, P. Kékicheff, J. Colloid Interface Sci. 2021, 582, 1158-1178. 
[44] L. Gazzera, R. Milani, L. Pirrie, M. Schmutz, C. Blanck, G. Resnati, P. Metrangolo, M. P. Krafft, Angew. Chemie 2016, 128, 10419-10423.

[45] S. E. Friberg, Chang Sup Wohn, B. Greene, R. Van Gilder, J. Colloid Interface Sci. 1984, $101,593-595$.

[46] S. E. Friberg, I. Blute, H. Kunieda, P. Stenius, Langmuir 1986, 2, 659-664.

[47] S. E. Friberg, I. Blute, P. Stenius, J. Colloid Interface Sci. 1989, 127, 573-582.

[48] E. Forel, B. Dollet, D. Langevin, E. Rio, Phys. Rev. Lett. 2019, 122, 088002.

[49] D. Langevin, Adv. Colloid Interface Sci. 2020, 275, 102075.

[50] H.-H.-Q. Dinh, E. Santanach-Carreras, M. Lalanne-Aulet, V. Schmitt, P. Panizza, F. Lequeux, Langmuir 2021, 37, 8726-8737.

[51] B. P. Binks, P. D. I. Fletcher, M. D. Haynes, Colloids Surfaces A Physicochem. Eng. Asp. 2003, 216, 1-8.

[52] Y. Ando, H. Tabata, M. Sanchez, A. Cagna, D. Koyama, M. P. Krafft, Langmuir 2016, 32, 12461-12467.

\section{TOC}

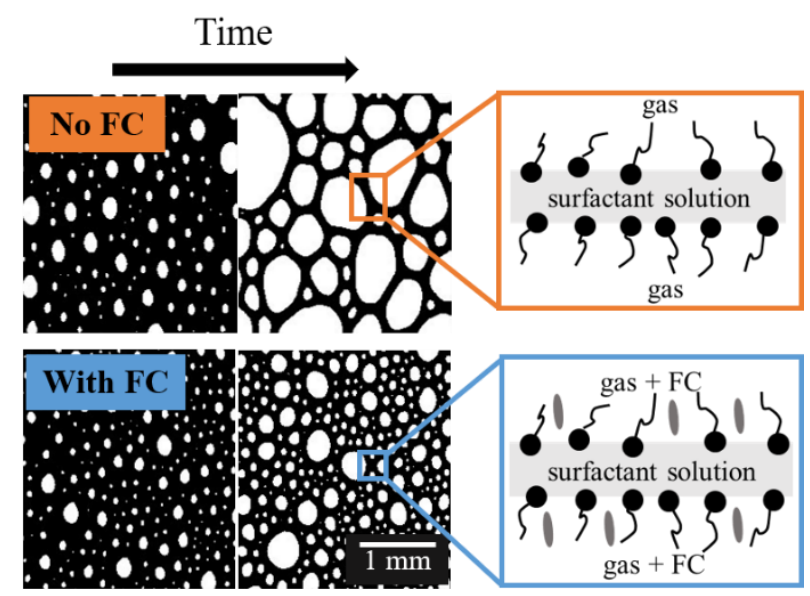

Tuning the stability of foams remains a major challenge for the optimization for applications. The use of co-adsorbates from the gas phase is a new possibility to control this stability. Here we show that fluorocarbon (FC) vapors not only prevent gas exchange between bubbles, as widely known, but also efficiently increase the stability of foam films against rupture. 
